\title{
Morphological Operators with Discrete Line Segments
}

\author{
Pierre Soille \\ EC Joint Research Centre, Space Applications Institute \\ TP 441, I-21038 Ispra, Italy \\ Pierre.Soille@jrc.it \\ http://ams.egeo.sai.jrc.it/soille
}

\begin{abstract}
The morphological approach to image processing consists in probing the image structures with a pattern of known shape called structuring element. In this paper, we concentrate on structuring elements in the form of discrete line segments, including periodic lines. We investigate fast algorithms, decomposition/cascade schemes, and translation invariance issues. Several application examples are provided.
\end{abstract}

\section{Introduction}

In mathematical morphology [15,20,21,9,23], image structures are extracted or filtered out by letting them interact with a pattern of known shape called structuring element. While discrete approximations of disk structuring elements are desirable in many applications, discrete line segments of a given length and orientation are best suited for processing thin structures such as roads in satellite images or ridges and valleys in fingerprints. In addition, cascades of morphological operators with line segments can be used for producing discrete approximations of disks. The goal of this paper is to study morphological operators based on discrete line segments.

The paper is organised as follows. Issues related to the definition of discrete line segments are detailed in Sec. 2. Recursive van Herk's algorithm for erosions and dilations is recalled in Sec. 3 together with its extension to arbitrary directions, grey scale structuring elements, and 3-dimensional images. Section 4 concentrates on cascades of morphological operators with line segments. Parallel combinations of a bank of openings and closings with line segments for varying orientations are investigated in Sec. 5. Before concluding, we show in Sec. 6 that binary and grey scale convex hulls can be obtained by performing max computations along a series of periodic lines.

\section{Discrete Line Segments}

\subsection{Connected Line Segments}

Rosenfeld [19] showed that a digital arc $L$ is a digitization of a straight line segment if and only if it satisfies the so-called chord property, i.e., the line segment

G. Borgefors, I. Nyström, and G. Sanniti di Baja (Eds.): DGCI 2000, LNCS 1953, pp. 78-98, 2000. (c) Springer-Verlag Berlin Heidelberg 2000 
joining any two points of $L$ lies everywhere within a distance 1 of $S$. Note that the Kim's area property [13] and chord property are equivalent as pointed out later by the same author [14].

Bresenham [2] has proposed an attractive algorithm for generating discrete lines satisfying the chord property (see [4] for further improvements). This algorithm is especially attractive on systems not equipped for fast floating point operations because it requires only integer arithmetic operations. The pixel selection criterion is based on a distance criterion. For instance, Fig. 1 shows a continuous line segment of slope $3 / 5$ over a square grid of pixels. The pixels belonging to the discrete line approximating this continuous line segment are determined using distance measurements along each vertical line linking the centres of the pixels of the grid. These vertical lines are represented by vertical dotted lines in Fig. 1. For each vertical line, the pixel whose centre is closest to the continuous line belongs to the corresponding discrete line. When the slope is larger than 1, the horizontal dotted lines linking the centres of the pixels must be considered instead of the vertical ones.

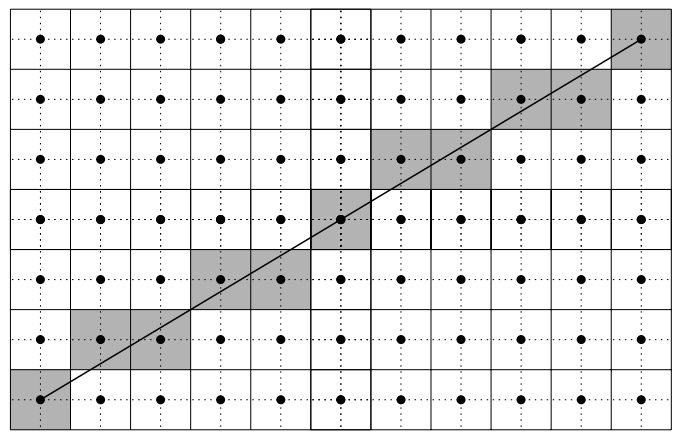

Fig. 1. Euclidean line of slope $3 / 5$ and corresponding Bresenham discrete line: each time the Euclidean line cuts the vertical lines linking the centres of the pixels (i.e., the vertical dotted lines), the centre of the pixel which is closest to the Euclidean line defines a pixel of the discrete line

Note that for a Bresenham line of slope in the form of an irreducible fraction $y / x$, the number of distinct line segments occurring along the discrete line equals $\max (|x|,|y|)$. It is convenient to include the forms $0 / 1$ and $1 / 0$ for referring to horizontal and vertical lines respectively. The number of distinct line segments corresponds to the periodicity $k$ of the elementary pattern occurring along the Bresenham line, i.e., $k=\max (|x|,|y|)$. In the sequel, we denote by $L_{\lambda_{i},(x, y)}$ the connected line segment obtained by considering $\lambda$ successive pixels of a Bresenham line of slope $y / x$, starting from the $i$ th pixel of the line $(i \in\{1, \ldots, k\})$. For example, Fig. 2 shows the five line segments of length equal to 11 pixels occurring for a slope of $3 / 5: L_{11_{1},(5,3)}, L_{11_{2},(5,3)}, L_{11_{3},(5,3)}, L_{11_{4},(5,3)}$, and $L_{11_{5},(5,3)}$. 


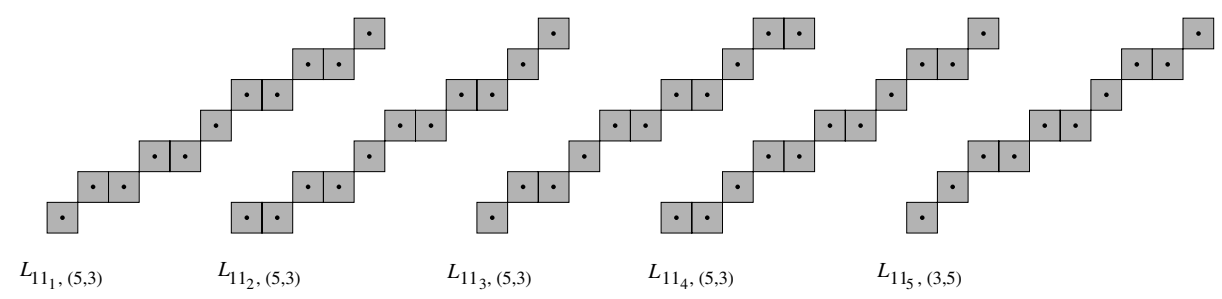

Fig. 2. The five possible line segments occurring along a Bresenham line of slope $3 / 5$ (here for a length of 11 pixels). Note that all these line segments satisfy the chord property

\subsection{Periodic Lines}

Digital connected line segments at arbitrary orientation are broad approximations of Euclidean line segments. This led Jones and Soille [11,12] to introduce the concept of periodic lines by considering only those points of the Euclidean line that fall exactly on grid points.

In mathematical terms, a periodic line $P_{\lambda, \boldsymbol{v}}$ is defined as follows:

$$
P_{\lambda, \boldsymbol{v}}=\bigcup_{i=0}^{i=\lambda-1} i \boldsymbol{v}
$$

where $\lambda>1$ is the number of points in the periodic line and $\boldsymbol{v}$ is a constant vector $^{1}$. The vector $\boldsymbol{v}$ is in the form $\boldsymbol{v}=(x, y)$ where $x, y \in \mathbf{Z}$. Similarly to Bresenham lines, we define the periodicity $k$ of the periodic line as follows: $k=\max (|x|,|y|)$. For example, Figs. 3a, b, and c correspond to the periodic lines $P_{3,(1,0)}, P_{3,(1,1)}$ and $P_{3,(2,1)}$ respectively.

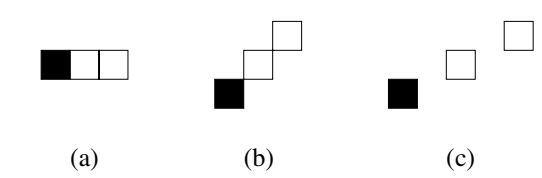

Fig. 3. Examples of periodic lines. (a) $P_{3,(1,0)}$. (b) $P_{3,(1,1)}$. (c) $P_{3,(2,1)}$. Points belonging to a line are indicated by squares. The position of the origin of each line is indicated by a black square

An interesting property of a Bresenham line of slope in the form of an irreducible fraction $y / x$ is that it is exactly covered by $\max (|x|,|y|)$ Euclidean lines

${ }^{1}$ Note that periodic lines were originally defined [12] as follows: $\cup_{i=0}^{i=\lambda} i \boldsymbol{v}$. Here, we have adapted the definition of periodic lines so that $\lambda$ equals to the number of pixels of the periodic line (rather than $\lambda+1$ in the original definition), in accordance with the parameter $\lambda$ used for Bresenham connected line segments $L$. 
of slope $y / x$. This property is illustrated in Fig. 4, for a line whose slope equals $2 / 3$. Indeed, due to the construction of a Bresenham line, the periodicity of the pixels in a line of slope $y / x$ equals $\max (|x|,|y|)$ and all these pixels belong to a Euclidean line of slope $y / x$. In other words, any Bresenham lines of slope $y / x$ is covered by $\max (|x|,|y|)$ periodic lines of the same slope. This property is at the basis of the translation invariant implementation of half-plane closings described in Sec. 6.
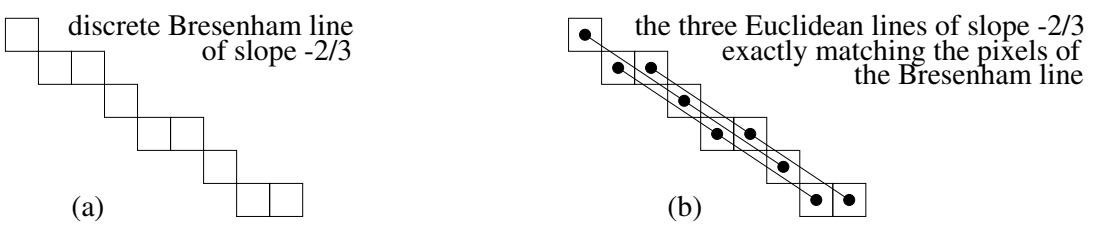

Fig. 4. (a) A Bresenham line segment of slope $-2 / 3$. (b) The three Euclidean line segments matching all points of $(a)$. Note that the intersection between each Euclidean line and the Bresenham line defines a periodic line $P_{3,(3,-2)}$

\subsection{Angular Resolution}

In a discrete grid, the angular resolution of a discrete line segment depends on its length. In a square grid, only $2 n-2$ directions can be defined with a connected line segment of odd length equal to $n$ pixels, and whose middle and extreme pixels are matched by the Euclidean line of the same orientation. By relaxing the condition regarding the middle pixel, there are $2(2 n-2)$ possible orientations for any $n \geq 2$. For example, Fig. 5 illustrates the 8 (reps. 16) possible orientations for a connected line segments containing 5 pixels.

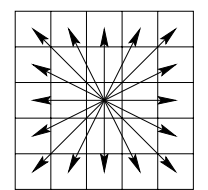

(a)

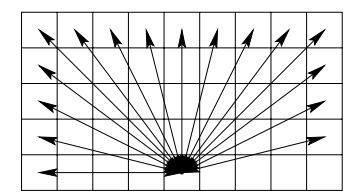

(b)

Fig. 5. (a) The 8 possible orientations for a line segment of 5 pixels and whose middle and extreme pixels are matched by the Euclidean line of the same orientation. (b) The 16 possible orientations by relaxing the condition concerning the middle pixel

If we merely impose to the line segments to contain at least two pixels exactly matching the corresponding Euclidean line, the number of distinct orientations 
equals $4 \operatorname{card}\left(F_{i}\right)-4$, where $F_{i}$ is the Farey sequence [3] of order $i$, and $\operatorname{card}(\cdot)$ returns the number of elements (i.e., cardinal number). The Farey sequence $F_{i}$ of order $i \geq 1$ is the ascending sequence of all fractions $p / q$ for which $0 \leq p / q \leq 1$, $q \leq i$, and $p$ and $q$ are nonnegative integers with no common divisors other than 1 (note that the form $0 / 1$ is included in the sequence). A graphical representation of all possible discrete slopes in the range $[0,1]$ for a line segment whose length is less than or equal to 32 pixels is shown in Fig. 6 .

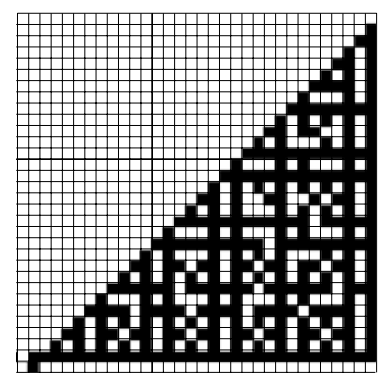

Fig. 6. Graphical representation of all possible discrete slopes in the range $[0,1]$ and for a length less than or equal to 32 pixels. Each slope is obtained by linking the origin (lower left pixel) to a given dark pixel

\section{Recursive Algorithm for Erosions and Dilations}

\subsection{Principle $[34,8]$}

The recursive algorithm is due to van Herk [34] and, almost simultaneously, Gil \& Werman [8] (see also [6]). We follow here the notations and description proposed in [23, pp. 77-78]. A 1-dimensional input image $f$ of length $n x$ is divided into blocks of size $\lambda$, where $\lambda$ is the length of the line segment in number of pixels. The elements of $f$ are indexed by indices running from 0 to $n x-1$. It is also assumed that $n x$ is a multiple of $\lambda$. Two temporary buffers $g$ and $h$ of length $n x$ are also required. In the case of dilation, the maximum is taken recursively inside the blocks in both the right and left directions (right for $g$ and left for $h$ ). When both $g$ and $h$ have been constructed, the result for the dilation $r$ at any coordinate $x$ is given by considering the maximum value between $g$ at position $x+\lambda-o-1$ and $h$ at position $x-o, o$ denoting the coordinate of the origin of the structuring element (e.g., 0 for the first pixel of the line segment). This recursive dilation algorithm can be written as follows: 


$$
\begin{aligned}
g(x) & =\left\{\begin{array}{l}
f(x), \quad \text { if } x=0, \lambda, \ldots,(m-1) \lambda, \\
\max [g(x-1), f(x)], \quad \text { otherwise. }
\end{array}\right. \\
h(x) & =\left\{\begin{array}{l}
f(x), \quad \text { if } x=m \lambda-1,(m-1) \lambda-1, \ldots, \lambda-1, \\
\max [h(x+1), f(x)], \quad \text { otherwise. }
\end{array}\right. \\
r(x) & =\max [g(x+\lambda-o-1), h(x+o)] .
\end{aligned}
$$

In [7], minor improvements to the recursive procedure are proposed. This is achieved by reducing the number of computations in the generation the forward and backward buffers and the merge procedure. Other enhancements for the simultaneous computation of min and max filters and openings/closings are also developed. When dealing with binary images, a recursive algorithm based on directional distance transforms also running in constant time is described in [17].

\subsection{Extension to Arbitrary Directions and Periodic Lines}

Principle $[26,27]$ The recursive procedure is directly applied to the image pixels falling along a line at given angle. The line is then translated and the whole procedure is repeated until all image pixels have been processed, i.e., until the translations of the line have swept the whole image plane. The direction of the translation depends on the slope of the line (see Fig. 7). By doing so, each

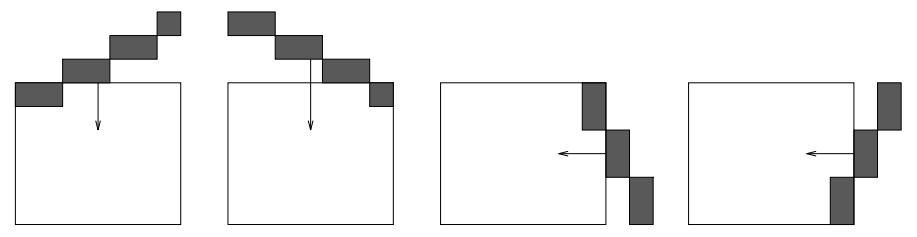

Fig. 7. Depending on its slope, the discrete line is drawn from an appropriate image corner. It is then translated in a unique direction in order to sweep the whole image plane while avoiding overlapping pixels (the arrows indicate the translation direction)

pixel is processed only once. When translating the line from its original position, the number of pixels falling within the image plane first increases, then remains constant, and finally decreases. If the image size along the translation direction is shorter than the distance between the extremity of the line falling off the image plane and the image plane, the constant zone is replaced by a zone where the line is increasing at one end while decreasing at the other. The number of pixels falling within the image after each translation can be efficiently updated using a run length coding of the discrete line. The algorithm is suited to line erosions and dilations in 3-dimensional images but requires a careful analysis of border effects. 
The procedure extends directly to periodic lines. Once the periodic structuring element has been defined, the discrete Bresenham line having the same orientation is traced from one of the image corners. Since the connected periodic line has the same orientation as the Bresenham line, the latter can be translated along the line so as to process all pixels of the line falling within the image plane. As described in the previous paragraph, the line is then translated in an appropriate direction and the procedure is repeated until the whole image plane has been swept. Figure 8 shows an example with the periodic line $P_{3,(2,1)}$.

Translation Invariance Issues As already noticed in [27], the shape of the connected line segment varies slightly from one pixel to another for all orientations not matching one of the principal directions of the digitisation grid. These variations are studied in detail in [31] while proposing solutions for achieving translation invariance wherever necessary. In general, there are $k$ possible outputs for a neighbourhood image operator $\Psi$ by a line segment of length $\lambda$ pixels applied along a Bresenham line of slope $y / x$ and sweeping the whole image definition domain. We denote them by $\Psi_{B L_{\lambda_{i}},(x, y)}$ where $i \in\{1, \ldots, k\}$ and $k=\max (|x|,|y|)$.

The recursive translation invariant strategy relies on the following structuring element decomposition:

$$
L_{n k_{i},(x, y)}=L_{k_{i},(x, y)} \oplus P_{n,(x, y)},
$$

where $\oplus$ denotes the Minkowski addition [16] and $i \in\{1, \ldots, k\}$. Therefore, the following relationships hold

$$
\begin{aligned}
& \delta_{L_{n k_{i},(x, y)}}=\delta_{L_{k_{i},(x, y)}} \delta_{P_{n,(x, y)}}, \\
& \varepsilon_{L_{n k_{i},(x, y)}}=\varepsilon_{L_{k_{i},(x, y)}} \varepsilon_{P_{n,(x, y)}} .
\end{aligned}
$$

We also show in [31] that the union (i.e., point-wise maximum $\vee$ for discrete grey scale images) of all $k$ possible non-TI openings is identical to the union of the openings by the $k$ possible line segments (the same result holds for closings $\phi$ by replacing the union with the point-wise minimum $\wedge$ ):

$$
\begin{aligned}
& \bigvee_{i=1}^{i=k} \gamma_{B L_{\lambda_{i}},(x, y)}=\bigvee_{i=1}^{i=k} \gamma_{L_{\lambda_{i}},(x, y)}, \\
& \bigwedge_{i=1}^{i=k} \phi_{B L_{\lambda_{i}},(x, y)}=\bigwedge_{i=1}^{i=k} \phi_{L_{\lambda_{i}},(x, y)} .
\end{aligned}
$$

\subsection{Extension to Grey Scale Structuring Elements}

The dilation of an image $f$ with a grey scale (also referred to as volumic or non-flat) structuring element $B_{v}$ is denoted by $\delta_{B_{v}}(f)$ and is defined as follows for each point $x$ :

$$
\left[\delta_{B_{v}}(f)\right](x)=\max _{b \in B_{v}}\left\{f(x+b)+B_{v}(b)\right\}
$$




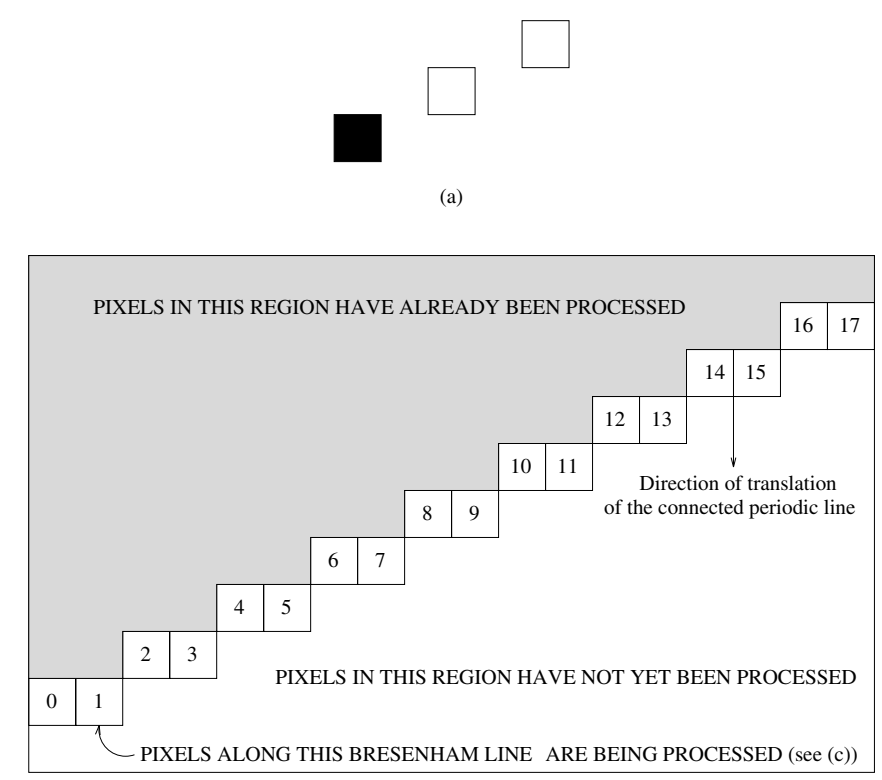

(b)

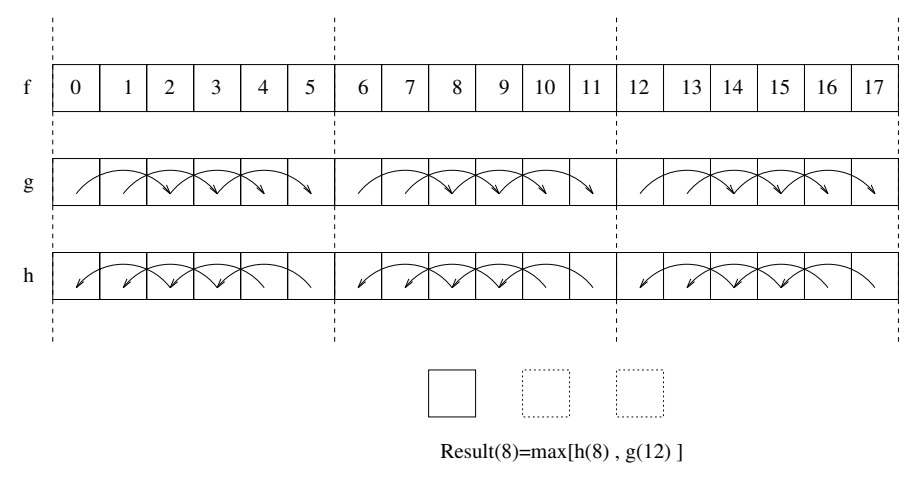

(c)

Fig. 8. Implementation of dilation with a periodic structuring element using a modified van Herk algorithm. (a) A periodic structuring element $P_{3,(2,1)}$, its origin being the black pixel. (b) The image plane after 9 translations of the corresponding Bresenham line. (c) Schematic of the algorithm: the value in $g$ or $h$ at the starting point of a cycle of arrowed arcs equals the original value in the input image $f$ (e.g., $g(6)=f(6)$ or $h(11)=f(11)$ ). The value at the pixel pointed by an arrowed arc equals the maximum between the value of $f$ at this position and the value at the beginning of the arrowed arc (e.g., $h(2)=\max [f(2), h(4)])$. This algorithm requires 3 max comparisons, whatever the number of pixels of the periodic line 
The grey scale weights of a grey scale structuring element should be set according to the image intensity values. The erosion is defined by duality with respect to set complementation: $\left[\varepsilon_{B_{v}}(f)\right](x)=\min _{b \in B_{v}}\left\{f(x+b)-B_{v}(b)\right\}$.

Grey scale structuring elements should be used with care because the corresponding erosions and dilations do not commute with scalings of the pixel intensity values $[18,29]$. Nevertheless, these structuring elements are useful for some applications such as the rolling-ball algorithm and the computation of shadows of an image seen as a topographic surface [32].

Let us show that the recursive procedure for computing $\min / \max$ filters (see Sec. 3.2) extends to grey scale periodic structuring elements, i.e., structuring elements whose domain of definition is a periodic line and whose grey scale values are defined as the index of the point $i$ in the periodic line multiplied by a real number $s$ defining the grey scale slope: $P(i \boldsymbol{v})=i s, \forall i \in\{0,1, \ldots, \lambda-1\}$ [28]. We denote by $P_{\lambda, \boldsymbol{v}, s}$ grey scale periodic structuring elements of grey scale slope s. Examples of grey scale periodic structuring elements are presented in Fig. 9.

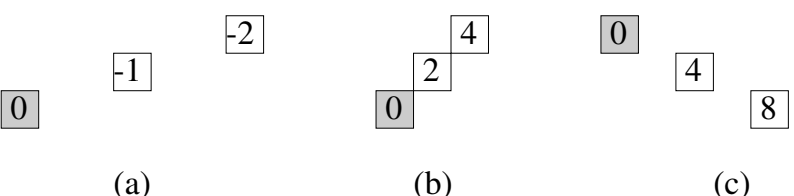

Fig. 9. Grey scale periodic structuring elements. (a) $P_{3,(3,1),-1}$. (b) $P_{3,(1,1), 2}$. (c) $P_{3,(2,-1), 4}$

The algorithm requires an additional buffer $f^{\prime}$. The values of the input image are copied in this buffer which is partitioned into blocks as described in Section 3.2. The periodic structuring element is then positioned at the first pixel of each block and the weights are added to each pixel of the block whose intersection with the structuring element is non-empty. The structuring element is then translated by one pixel to the right and the procedure is repeated until a weight has been added to all pixels of $f^{\prime}$ (there are $k-1$ translations per block). Once the values of the buffer $f^{\prime}$ have been calculated, the buffers $g$ and $h$ are computed from the buffer $f^{\prime}$ using the recursive procedure detailed in Section 3.2. Finally, the resulting value at each position equals the maximum value between the value in the buffer $h$ at the current position and the value in the buffer $g$ at the current position plus $x+k(\lambda-1)$. However, in order to ensure that the same weights are used for all positions, appropriate multiples of the slope $s$ must be added to $g$ and subtracted from $h$ beforehand. More precisely, $x \bmod \lambda$ times $s$ must be removed from $h$ and $(\lambda-1)-(x+\lambda-1) \bmod \lambda$ times $s$ must be added to $g$. For clarity and conciseness, the case of a connected periodic line of $\lambda$ pixels (i.e., the periodicity $k$ is equal to 1 ) with the origin matching the first pixel of 
the periodic line (i.e., $o=0)$ is presented hereafter:

$$
\begin{aligned}
f^{\prime}(x) & =f(x)+(x \bmod \lambda) s \\
g(x) & = \begin{cases}f^{\prime}(x) & \text { if } x \bmod \lambda=0, \\
\max \left[g(x-1), f^{\prime}(x)\right] & \text { otherwise. }\end{cases} \\
h(x) & = \begin{cases}f^{\prime}(x) & \text { if }(x+1) \bmod \lambda=0, \\
\max \left[h(x+1), f^{\prime}(x)\right] & \text { otherwise. }\end{cases} \\
r(x) & =\max [g(x+\lambda-1)+((\lambda-1)-(x+\lambda-1) \bmod \lambda) s, h(x)-(x \bmod \lambda) s] .
\end{aligned}
$$

Note that all divisions are integer divisions. This algorithm adapts directly to non-unitary periodicities (i.e., $k>1$ ). An example is given in Fig. 10 for the periodic line $P_{3,(2,0), s}$. For conciseness, we use a structuring element of 3 pixels only. Since our algorithm requires 3 max comparisons per pixel whatever the number of pixels in the structuring element, speed gains are obtained for larger structuring elements.

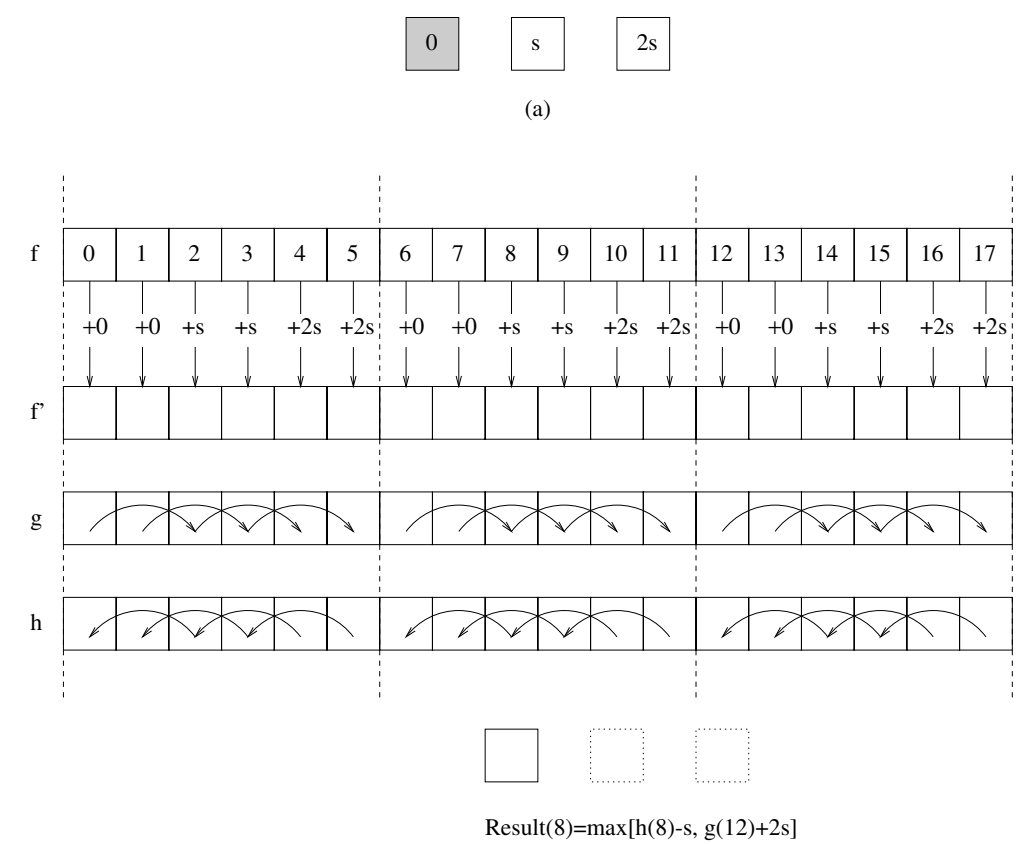

(b)

Fig. 10. Recursive $\min / \max$ filter with a grey scale periodic structuring element $P_{3,(2,0), s}$ (shaded origin). The structuring element is shown in (a) and the schematic of the algorithm in (b) 


\subsection{Extension to 3-Dimensional Images}

This extension is trivial in the sense that the very nature of the algorithm is 1-dimensional. In practice however, the implementation of a sweeping procedure allowing for the processing of the image volume along discrete lines while avoiding processing a voxel more than once requires a very careful analysis of the translation directions and handling of the image borders. The original position (image corner) of the line and the two translations to consider depend on the orientation of the line (there are 16 cases).

\section{Line Segment Cascades}

By cascading two erosions (resp. dilations) with vertical and horizontal line segments, one achieves erosions (resp. dilations) with square structuring elements:

$$
\square_{n}=L_{n,(1,0)} \oplus L_{n,(0,1)},
$$

where $\square_{n}$ is a square a width $n$ pixels. It has long been know that discrete diamond-shaped structuring elements cannot be generated by cascading erosions (resp. dilations) with structuring elements at 45 and -45 degrees. Although logarithmic decompositions have been proposed [33] for speeding up operations with diamond-shaped sets, the following simple and efficient decomposition can be used instead:

$$
\diamond_{n}=L_{n-1,(1,1)} \oplus L_{n-1,(1,-1)} \oplus \diamond_{2},
$$

where $\diamond_{n}$ is the diamond-shaped structuring element with a side of $n$ pixels $(n \geq$ 2 ), i.e., $\diamond_{2}$ is the 4 -connected neighbourhood plus its central pixel. By definition, $\diamond_{1}$ is a single pixel. An example is shown in Fig. 11. All these decompositions

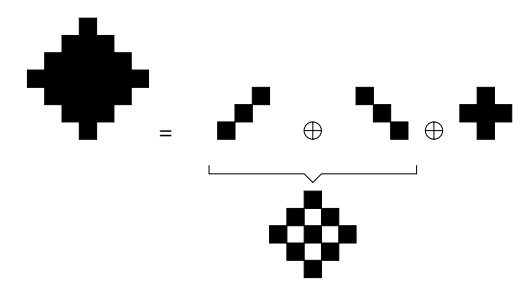

Fig. 11. Decomposition of a diamond-shaped structuring element of a width of 4 pixels using Eq. 9

substantially speed up operations for $n$ large enough (6 (resp. 10) $\mathrm{min} / \mathrm{max}$ comparisons per pixel whatever the width $n$ of the square (resp. diamond) when using the recursive algorithm instead of a number in $O\left(n^{2}\right)$ for the brute force algorithm). Note also that cascades of erosions/dilations by $\diamond_{2}$ currently used 
for generating operations with diamond-shaped structuring elements of larger size are not only less efficient than Eq. 9 but also only lead to diamonds of odd width. In the sequel of this section, we concentrate on cascades starting from periodic lines and leading to connected lines and discrete disks.

\subsection{Leading to Connected Lines}

Cascades of periodic lines can be used for generating connected line segments and granulometric families of disks. This idea has originally been developed in [12]. We summarise here the main results. It is apparent from the example shown in Fig. 3c that in general a periodic line is not connected. In fact, in the twodimensional case using 8-connectivity, the only examples of connected periodic lines are when both the horizontal and vertical components of the periodicity are either -1 , 0 , or 1 . However, periodic lines can be cascaded with other periodic lines or carefully chosen structuring elements to form a connected line (this principle it at the very basis of Eq. 2). Indeed, if $P_{\lambda, \boldsymbol{v}}$ is a periodic line, where $\lambda>1$, and $A$ is any connected line with end points given by $\mathbf{0}$ and $\boldsymbol{v}$, then $L_{\lambda}=$ $A \oplus P_{\lambda, \boldsymbol{v}}$ is a connected line with the same end points as $P_{\lambda, \boldsymbol{v}}$. An example is shown in Fig. 12 using the periodic line $P_{3,(2,1)}$ from Fig. 3c. In Fig. 12a are

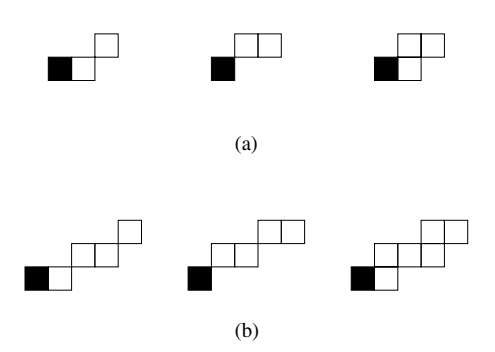

Fig. 12. Connected periodic lines. (a) Examples of the connected line $A$ for the periodicity $(2,1)$. (b) Corresponding connected periodic lines $L_{2}=A \oplus P_{2,(2,1)}$

examples of the line $A$ that have the two end points $(0,0)$ and $(2,1)$. Note that the line $A$ may be of any type so long as it is connected; the first two we show here are 8-connected Bresenham lines and the third is 4-connected. Although it would be advantageous if the connected line $A$ was also periodic, as this would admit a fast and translation-invariant implementation of $A$, this is only possible for a restricted class of periodicities. The corresponding connected periodic lines, given by the cascade $A \oplus P_{2,(2,1)}$, are shown below each line $A$ in Fig. 12b.

The granulometric properties of periodic shapes are summarised by the following theorem [12]: Given any set $A$ and periodic shape $S_{\boldsymbol{\lambda}, \mathbf{v}}$, the opening $\Gamma_{\boldsymbol{\lambda}}(f)=\gamma_{A \oplus S_{\boldsymbol{\lambda}, \mathbf{V}}}(f)$ admits $\Gamma_{\boldsymbol{\lambda}+\boldsymbol{\mu}} \leq \Gamma_{\boldsymbol{\lambda}}$, where $\boldsymbol{\lambda}, \boldsymbol{\mu} \geq \mathbf{0}$. It is therefore a granulometric function with size vector $\lambda$.

A particular application is to linear granulometries. Since the line $P_{\lambda, \boldsymbol{v}}$ is an example of a periodic shape $S_{\boldsymbol{\lambda}, \mathbf{v}}$, we may state the following: If $\Gamma_{\lambda}(f)=\gamma_{L_{\lambda}}(f)$, 
where $L_{\lambda}$ is a connected periodic line, then $\Gamma_{\lambda}$ is a granulometric function with size parameter $\lambda>1$. Figure 13 shows an example using the periodic line $P_{3,(2,1)}$ from Figs. 3 and 12. Figure $13 \mathrm{a}$ is a connected line $A$ with end points $(0,0)$ and $(2,1)$. Figures $13 \mathrm{a}, \mathrm{b}$ and $\mathrm{c}$ respectively are the connected periodic lines $A \oplus P_{1,(2,1)}, A \oplus P_{2,(2,1)}$ and $A \oplus P_{3,(2,1)}$ which are used to generate the first three members of the family of the granulometric function $\Gamma_{\lambda}(f)=\gamma_{L_{\lambda}}(f)$. Note

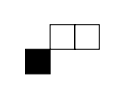

(a)

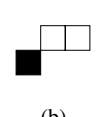

(b)

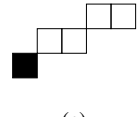

(c)

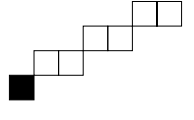

(d)

Fig. 13. Granulometric functions can be generated from a connected periodic line. (a) Connected line A. (b), (c) and (d) Growth of the connected periodic line

that the increase in length of $L_{\lambda}$ equals $k$ pixels, the periodicity of the underlying periodic line. By considering the union of openings described in Sec. 3.2, one can however increase the length of the structuring element one pixel at a time while satisfying all axioms of a granulometry. The resulting directional granulometry $\Gamma$ for a given slope $y / x$ and size parameter $\lambda$ is then defined as follows:

$$
\Gamma_{\lambda,(x, y)}=\bigvee_{i=1}^{i=k} \gamma_{L_{\lambda_{i},(x, y)}} .
$$

\subsection{Leading to Discrete Disks}

In Euclidean morphology, Matheron [15, p. 94] has shown that for $\theta_{1}, \theta_{2}, \ldots \theta_{n}$ distinct in $[0, \pi), k_{1}, k_{2}, \ldots, k_{n}>0$, then $k_{1} L_{\theta_{1}} \oplus k_{2} L_{\theta_{2}} \oplus \cdots \oplus k_{n} L_{\theta_{n}}$ is a convex polygon of $2 n$ sides whose opposite edges are of length $2 k_{i}$ and have orientation given by $\theta_{i}$. Adams [1] used this principle for generating disks (and spheres) of increasing size from cascades of dilations by discrete Bresenham line segments. In [12], it has been show that cascades of periodic lines lead to better results in the sense that the resulting disks are symmetric. The approximation of a Euclidean disk (ball) $B$ can be written as follows: $B \approx S_{\boldsymbol{\lambda}, \boldsymbol{v}}$. However, no clue was given on how to select the vector of sizes and the corresponding vector of periodic lines. This can be achieved as follows. Suppose we look for the best discrete approximation of an Euclidean disk using cascades of line segments whose Euclidean length equals $l$. We then consider Fig. 6 and look for all slopes defined for this length and use the corresponding periodic lines with the maximal number of pixels so that the corresponding Euclidean line segment does not exceed $l$. We denote by $B_{l}$ the corresponding approximation. For example we 
have the following cascades for the five first approximations:

$$
\begin{aligned}
B_{1}= & P_{2,(1,0)} \oplus P_{2,(0,1)}, \\
B_{\sqrt{2}}= & P_{2,(1,0)} \oplus P_{2,(0,1)} \oplus P_{2,(1,1)} \oplus P_{2,(1,-1)}, \\
B_{2}= & P_{3,(1,0)} \oplus P_{3,(0,1)} \oplus P_{2,(1,1)} \oplus P_{2,(1,-1)}, \\
B_{\sqrt{5}}= & P_{3,(1,0)} \oplus P_{3,(0,1)} \oplus P_{2,(1,1)} \oplus P_{2,(1,-1)} \\
& \oplus P_{2,(2,1)} \oplus P_{2,(2,-1)} \oplus P_{2,(1,2)} \oplus P_{2,(1,-2)}, \\
B_{2 \sqrt{2}}= & P_{3,(1,0)} \oplus P_{3,(0,1)} \oplus P_{3,(1,1)} \oplus P_{3,(1,-1)} \\
& \oplus P_{2,(2,1)} \oplus P_{2,(2,-1)} \oplus P_{2,(1,2)} \oplus P_{2,(1,-2)} .
\end{aligned}
$$

Figure 14 displays the eight first disks. Notice that, by construction, these disks

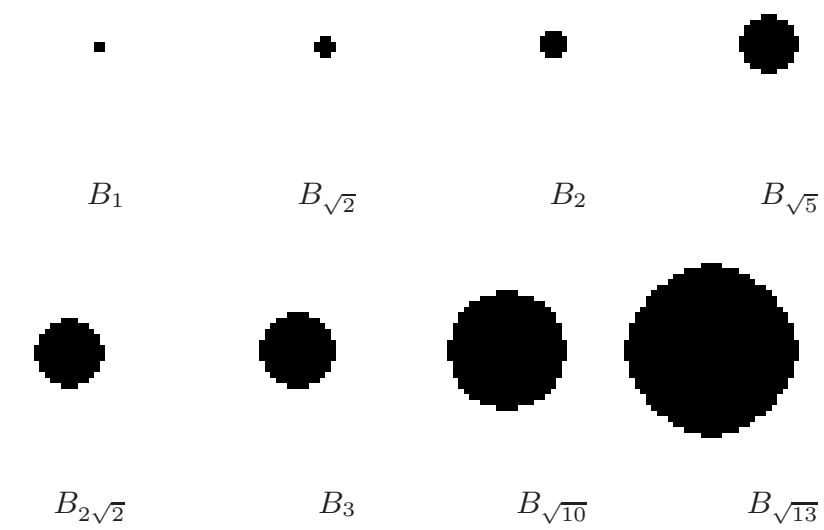

Fig. 14. Radial decompositions of discrete disks of increasing size using cascades of dilations with periodic lines. Each disk is symmetric, convex (see Sec. 6), and is opened for all disks of smaller size: $\gamma_{B_{i}}\left(B_{j}\right)=\emptyset$ for all $i \leq j$ (see text for the definition of each $B_{i}$ )

form a granulometry with size parameter given by $l$. In contrast, radial decompositions using Bresenham lines [1] cannot be used to generate a granulometric function, neither the disks obtained by thresholding the Euclidean distance computed from a center pixel (the first element would be $\diamond_{2}$ and the second $\square_{3}$ but it is not opened by the first, i.e., the absorption property is not satisfied). The generation of a granulometric family of discrete spheres $S$ from cascades of periodic lines can be achieved using a similar approach. For example, we have the following two first decompositions:

$$
\begin{aligned}
S_{1}= & P_{2,(1,0,0)} \oplus P_{2,(0,1,0)} \oplus P_{2,(0,0,1)}, \\
S_{\sqrt{2}}= & S_{1} \oplus P_{2,(1,1,0)} \oplus P_{2,(1,-1,0)} \\
& \oplus P_{2,(0,1,1)} \oplus P_{2,(0,1,-1)} \oplus P_{2,(1,0,1)} \oplus P_{2,(1,0,-1)} .
\end{aligned}
$$




\section{Opening/Closings by Line Segments}

\subsection{Basics}

Directional openings and closings are useful for a wide range of practical applications such as fingerprint and fibre analysis, document interpretation (where thin lines have to be tracked), industrial inspection (where cracks, scratches, and other elongated defects have to be detected), and satellite imagery for the detection of road networks. A bank of directional openings and/or closings can also be used for extracting long thin objects of an image. This approach is illustrated in Fig. 15 for the extraction of bus-like structures in a 1 metre resolution satellite images. Other applications of directional morphological filters are detailed in [25] for the processing of veins appearing on plant leaves and [35] for the filtering of laid lines appearing in paper watermarks. When the elongated structures contains gaps, better results are obtained by considering rank-max directional openings [31]. The recursive algorithm described in Sec. 3 is then replaced by the moving histogram algorithm $[5,10]$ suited for rank filters.

\subsection{Orientation Field}

The orientation at a given pixel $x$ in an image is defined as the orientation of the line segment that minimises the difference between the grey-level value in the original image at $x$ and the grey-level value at the same location in the image filtered by the considered line segment [30,31]. Openings should be used for image structures that are brighter than their background (i.e., 'positive' image structures) and closings for image structures darker than their background (i.e., 'negative' image structures).

In mathematical terms, we define the positive orientation at a given image pixel $x$ and for a given scale $\lambda$ as the orientation of the directional morphological opening of length $\lambda$ which modifies the least the original image value at position $x$. We denote the the positive orientation by $\mathrm{Dir}^{+}$, the negative orientation Dir- being defined by duality:

$$
\begin{aligned}
& \operatorname{Dir}_{\lambda}^{+}(f)(x)=\left\{\theta_{i} \mid \gamma_{L_{\lambda, \theta_{i}}}(f)(x) \geq \gamma_{L_{\lambda, \theta_{j}}}(f)(x), \forall \theta_{i} \neq \theta_{j}\right\}, \\
& \operatorname{Dir}_{\lambda}^{-}(f)(x)=\left\{\theta_{i} \mid \phi_{L_{\lambda, \theta_{i}}}(f)(x) \leq \phi_{L_{\lambda, \theta_{j}}}(f)(x), \forall \theta_{i} \neq \theta_{j}\right\} .
\end{aligned}
$$

The positive (resp. negative) directional signature at a given pixel can be obtained by plotting the normalised opened (resp. closed) values versus the orientation of the line segment. This signature can then be used to detect crossing lines, flat zones, etc. We also define the following quantities for each point $x$ of the input image $f$ :

$$
\begin{aligned}
\operatorname{Max}_{\lambda}^{+}(f)(x) & =\left\{\gamma_{L_{\lambda, \theta_{i}}}(f)(x) \mid \gamma_{L_{\lambda, \theta_{i}}}(f)(x) \geq \gamma_{L_{\lambda, \theta_{j}}}(f)(x), \forall \theta_{i} \neq \theta_{j}\right\}, \\
\operatorname{Min}_{\lambda}^{+}(f)(x) & =\left\{\gamma_{L_{\lambda, \theta_{i}}}(f)(x) \mid \gamma_{L_{\lambda, \theta_{i}}}(f)(x) \leq \gamma_{L_{\lambda, \theta_{j}, \lambda}}(f)(x), \forall \theta_{i} \neq \theta_{j}\right\}, \\
\operatorname{Gdir}_{\lambda}^{+}(f)(x) & =\operatorname{Max}_{\lambda}^{+}(f)(x)-\operatorname{Min}_{\lambda}^{+}(f)(x) .
\end{aligned}
$$




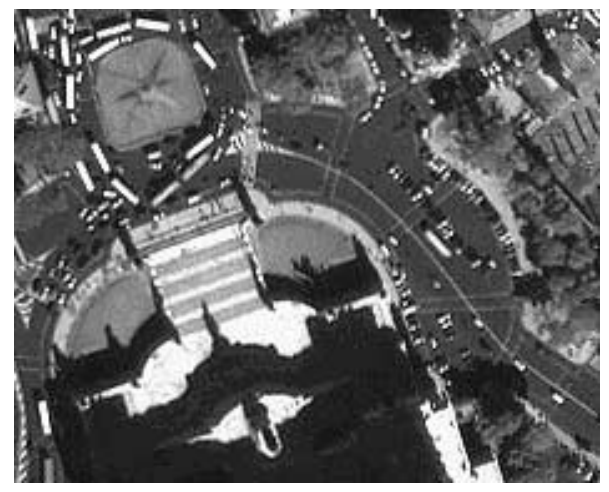

(a) Input satellite image (IKONOS) showing Piazza Venetia in Roma

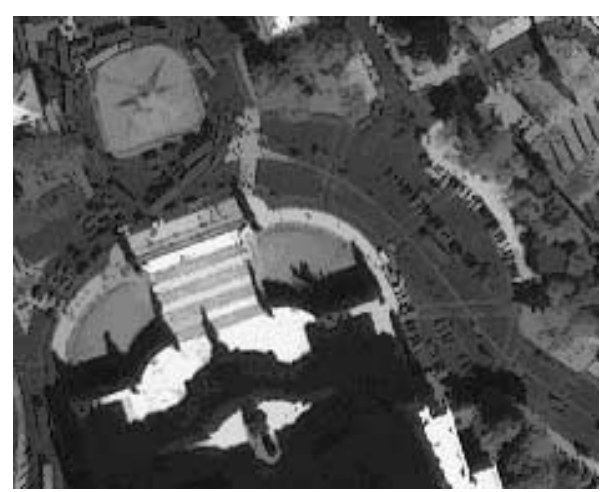

(c) Union of openings by line segments slightly longer than buses (followed by reconstruction of original image)

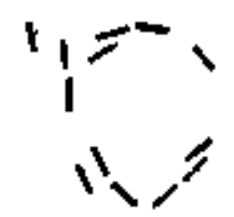

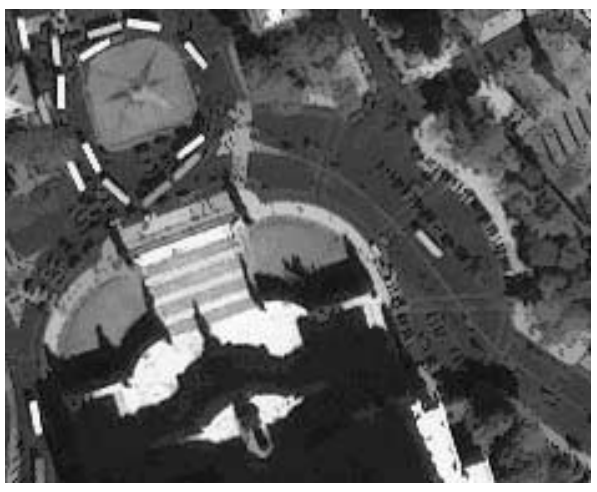

(b) Union of openings by line segments slightly shorter than buses (followed by reconstruction of original image)

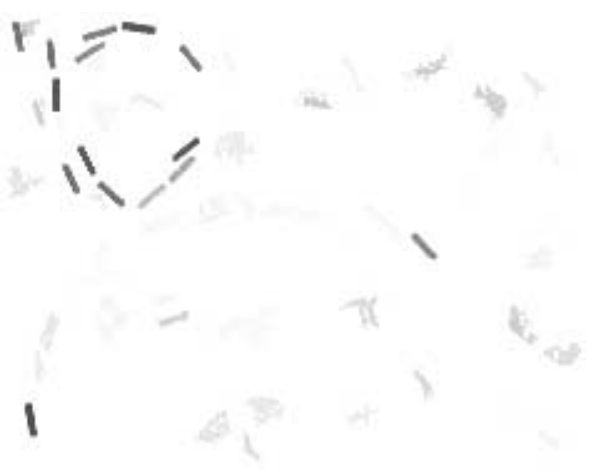

(d) Difference between image (b) and (c)

\section{1}

(e) Global threshold of (d)

Fig. 15. Extraction of bus-like structures appearing in a 1 metre resolution satellite image (IKONOS) using union of openings by line segments 
$\mathrm{Gdir}_{\lambda}^{+}$can be interpreted as the strength of the positive orientation: it will output a small value if there is no predominant orientation for a structuring element length of $\lambda$ pixels. $\operatorname{Max}_{\lambda}^{-}, \operatorname{Min}_{\lambda}^{-}$, and $\operatorname{Gdir}_{\lambda}^{-}$are defined by replacing the opening with the closing in Eqs. 13-14.

When comparing the values of $\mathrm{Gdir}^{+}$and $\mathrm{Gdir}^{-}$of a given pixel, it is possible to detect whether it belongs to a positive or negative image structure: positive, if $\mathrm{Gdir}^{+}>\mathrm{Gdir}^{-}$, negative, otherwise. We denote by Gdir the point-wise maximum between the images $G \operatorname{dir}^{+}$and $G_{d i r}^{-}: G d i r=G d i r^{+} \vee G d i r^{-}$. The image of directions Dir is then defined as follows:

$$
\operatorname{Dir}_{\lambda}(f)(x)=\left\{\begin{array}{l}
\operatorname{Dir}_{\lambda}^{+}(f)(x), \text { if } \operatorname{Gdir}_{\lambda}(f)(x)=\operatorname{Gdir}_{\lambda}^{+}(f)(x), \\
\operatorname{Dir}_{\lambda}^{-}(f)(x), \text { otherwise. }
\end{array}\right.
$$

A colour representation of the local orientation information is then simply achieved by equating the orientation information (i.e., either $\operatorname{Dir}^{+}, \mathrm{Dir}^{-}$, or Dir) to the hue and strength of the orientation information (i.e., either $\mathrm{Gdir}^{+}$, Gdir $^{-}$, or Gdir) to the lightness component of the image, the colours being fully saturated in all cases. For example, the colour representation of the local orientation by opening is illustrated in Fig. 16 on IRS satellite image of Athens.

\section{Closing by a Half-Plane and Convex Hulls}

In [22], we have been shown that the convex hull transformation can be defined in terms of an intersection (point-wise minimum $\wedge$ ) of half-plane closings. Hence, denoting by $\phi$ the closing transformation, $\pi_{\theta}$ a closed half-plane having a given slope $\theta=\arctan (y / x)$, and $\check{\pi}_{\theta}$ the reflected half-plane, the convex hull transformation $\mathrm{CH}$ of a grey scale image $f$ is defined as follows:

$$
C H(f)=\bigwedge_{\theta}\left[\phi_{\pi_{\theta}}(f) \wedge \phi_{\check{\pi}_{\theta}}(f)\right] .
$$

In the discrete case and for a bounded image, only a finite number of directions need to be considered. More precisely [24], let us first define the convex hull $\mathrm{CH}_{0}$ of order 0 as the intersection of the horizontal and vertical half-planes (i.e., slopes in the form $0 / 1$ and $1 / 0$ ). The convex hull of order 0 is nothing but the strong convex hull in the 4 -connected graph. The convex hull $C H_{i}$ of order $i \in \mathbf{N}$ is then defined as the intersection of all half-planes whose slopes are in the form of $y / x$ where $x$ and $y$ are integers in the range $[-i, i]$ with no common divisors other than 1 . The corresponding number of slopes is given by $4 \operatorname{card}\left(F_{i}\right)-4$ (see also Sec. 2.3). Since the orientations considered for any order $i \geq 0$ is a subset of the orientations considered for the order $i+1$, the following ordering relationship is satisfied: $\mathrm{CH}_{i+1} \leq \mathrm{CH}_{i}$.

For a $n \times n$ image, convergence is reached at the latest for the order $n-1$ (upper bound). The actual order number depends on the shape of the image objects. In practice, the number of half-plane closings corresponding to the upper bound may be too large for applications where speed is an issue. In this case, 

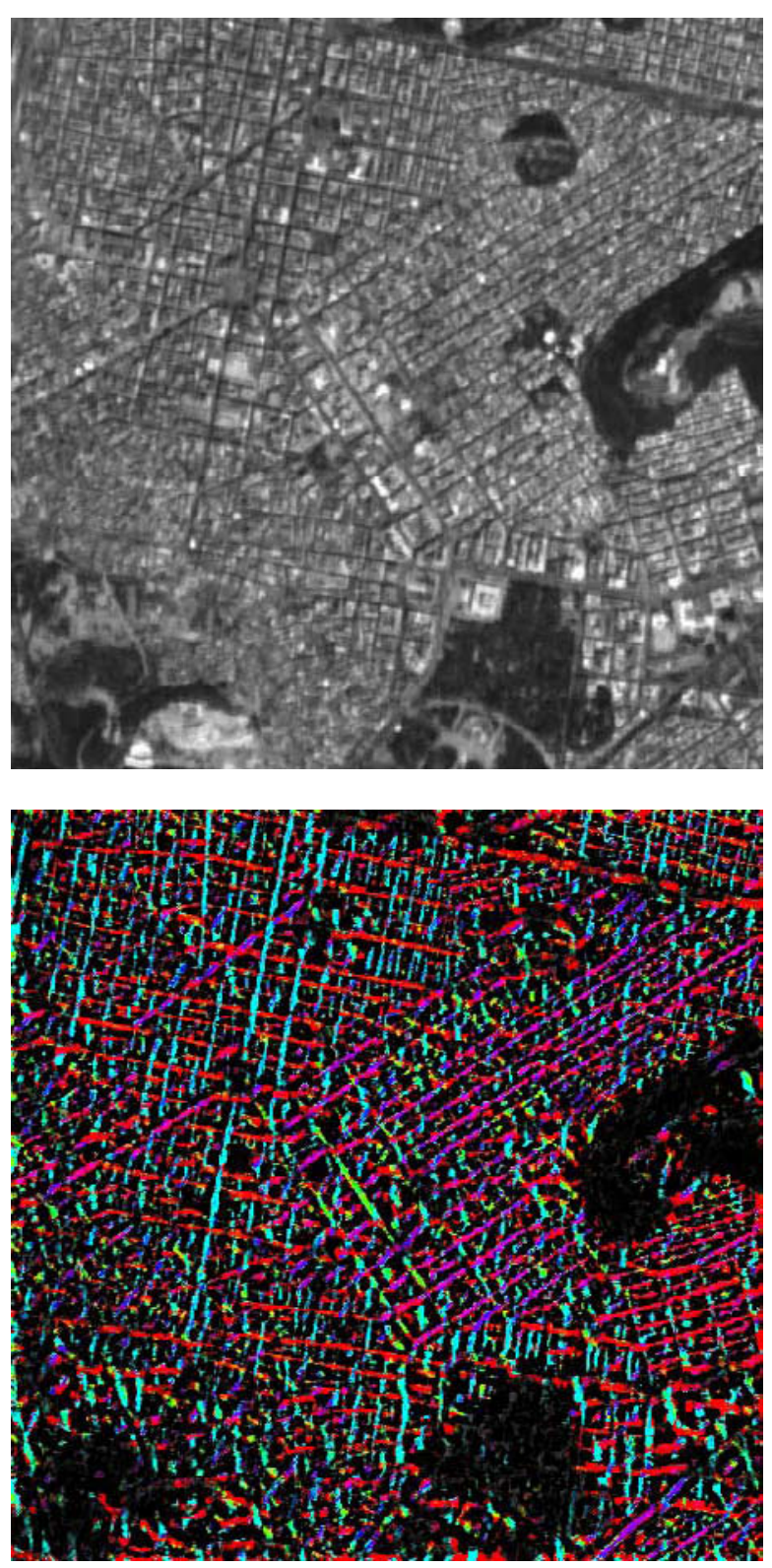

Fig. 16. Image of the local orientation enhancing the road network of a city. Top: input IRS image of Athens. Bottom: colour representation of $\mathrm{Dir}^{-}$. The structuring element used in this experiment is a line segment of 11 pixels using the moving histogram TI implementation and a rank of 4 for the corresponding rank-max openings 
a trade-off between accuracy and computation time must be considered. For example, a convex approximation of the discrete convex hull of order $n \geq 1$ is achieved by performing half-plane closings whose slopes are in the form $\pm i / n$ and $\pm n / i$, where $i \in\{0, \ldots, n\}$ (there are only $4 n-4$ such slopes). We denote by $\widetilde{C H}_{n}$ the corresponding approximation. Notice that the following ordering relation holds: $C H_{n} \leq \widetilde{C H_{n}}$. In [24], we show that if $C H_{w}$ outputs the exact discrete convex hull (i.e., $\mathrm{CH}=\mathrm{CH}$ ), then $\widetilde{\mathrm{CH}}_{o>w} \backslash \mathrm{CH}$ is at most one-pixel thick.

Finally, note that the translation-invariant implementation of half-plane closings can be achieved by processing the pixels in the order they are reached when progressively translating each Euclidean half-plane $\pi_{\theta}$ so as to sweep the whole image definition domain. By doing so, the pixels reached at any given step correspond to those falling along the periodic line having the same slope as the half-plane.

\section{Conclusion}

The study of discrete line segments for morphological operators leads to a wide variety of developments of interest to both discrete geometry and image analysis. The resulting structuring elements are useful not only for filtering thin image structures but also for generating sound approximations of discrete disks and grey scale convex hulls.

\section{References}

1. R. Adams. Radial decomposition of discs and spheres. Computer Vision, Graphics, and Image Processing: Graphical Models and Image Processing, 55(5):325-332, September 1993. 90, 91

2. J. Bresenham. Algorithm for computer control of digital plotter. IBM System Journal, 4:25-30, 1965. 79

3. J. Farey. On a curious property of vulgar fractions. Philosophical Magazine, 1816. See http://cut-the-knot.com/blue/FareyHistory.html for a historical note and a copy of Farey's original letter. 82

4. J. Foley, A. van Dam, S. Feiner, and J. Hughes. Computer graphics - Principles and Practice-. Addison-Wesley, 2nd edition, 1990. 79

5. G. Garibotto and L. Lambarelli. Fast on-line implementation of two dimensional median filtering. Electronic Letters, 15(1):24-25, January 1979. 92

6. D. Gevorkian, J. Astola, and S. Atourian. Improving Gil-Werman algorithm for running Min and Max filters. IEEE Transactions on Pattern Analysis and Machine Intelligence, 19(5):526-529, May 1997. 82

7. J. Gil and R. Kimmel. Efficient dilation, erosion, opening, and closing algorithms. In J. Goutsias, L. Vincent, and D. Bloomberg, editors, Mathematical Morphology and Its Applications to Image and Signal Processing, volume 18 of Computational Imaging and Vision, pages 301-310, Boston, 2000. Kluwer Academic Publishers. Proc. of ISMM'2000, Palo Alto, June 26-29. 83 
8. J. Gil and M. Werman. Computing 2-D min, median, and max filters. IEEE Transactions on Pattern Analysis and Machine Intelligence, 15(5):504-507, May 1993. 82

9. H. Heijmans. Morphological image operators. Advances in Electronics and Electron Physics. Academic Press, Boston, 1994. 78

10. T. Huang, G. Yang, and G. Tang. A fast two-dimensional median filtering algorithm. IEEE Transactions on Acoustics, Speech, and Signal Processing, 27(1):1318, February 1979. 92

11. R. Jones and P. Soille. Periodic lines and their applications to granulometries. In P. Maragos, W. Schafer, and M. Butt, editors, Mathematical Morphology and its Applications to Image and Signal Processing, pages 264-272. Kluwer Academic Publishers, 1996. Proc. of ISMM'96. 80

12. R. Jones and P. Soille. Periodic lines: Definition, cascades, and application to granulometries. Pattern Recognition Letters, 17(10):1057-1063, September 1996. $80,89,90$

13. C. Kim. On the cellular convexity of complexes. IEEE Transactions on Pattern Analysis and Machine Intelligence, 3:617-625, 1981. 79

14. C. Kim. On cellular straight line segments. Computer Graphics and Image Processing, 18(4):369-381, April 1982. 79

15. G. Matheron. Random sets and integral geometry. Wiley, New York, 1975. 78, 90

16. H. Minkowski. Über die Begriffe Länge, Oberfläche und Volumen. Jahresbericht der Deutschen Mathematiker Vereinigung, 9:115-121, 1901. 84

17. D. Nadadur and R. Haralick. Recursive binary dilation and erosion using digital line structuring elements in arbitrary orientations. IEEE Transactions on Image Processing, 9(5):749-759, May 2000. 83

18. J.-F. Rivest, J. Serra, and P. Soille. Dimensionality in image analysis. Journal of Visual Communication and Image Representation, 3(2):137-146, 1992. 86

19. A. Rosenfeld. Digital straight line segments. IEEE Transactions on Computers, 23:1264-1269, 1974. 78

20. J. Serra. Image analysis and mathematical morphology. Academic Press, London, 1982. 78

21. J. Serra, editor. Image analysis and mathematical morphology. Volume 2: Theoretical advances. Academic Press, London, 1988. 78

22. P. Soille. Grey scale convex hulls: definition, implementation, and application. In H. Heijmans and J. Roerdink, editors, Mathematical Morphology and its Applications to Image and Signal Processing, volume 12 of Computational Imaging and Vision, pages 83-90. Kluwer Academic Publishers, Dordrecht, 1998. Proc. of ISMM'98. 94

23. P. Soille. Morphological Image Analysis. Springer-Verlag, Berlin, Heidelberg, New York, 1999. 78, 82

24. P. Soille. From binary to grey scale convex hulls. Fundamenta Informaticae, 41(12):131-146, January 2000. 94, 96

25. P. Soille. Morphological image analysis applied to crop field mapping. Image and Vision Computing, 18(13), October 2000. 92

26. P. Soille, E. Breen, and R. Jones. A fast algorithm for $\min / \max$ filters along lines of arbitrary orientation. In I. Pitas, editor, Proc. of 1995 IEEE Workshop on Nonlinear Signal and Image Processing, pages 987-990, Neos Marmaras, June 1995. 83

27. P. Soille, E. Breen, and R. Jones. Recursive implementation of erosions and dilations along discrete lines at arbitrary angles. IEEE Transactions on Pattern Analysis and Machine Intelligence, 18(5):562-567, May 1996. 83, 84 
28. P. Soille and R. Jones. Periodic lines: Fast implementation and extensions to greyscale structuring elements and 3-D images. Technical report, Fraunhofer IPK (Berlin)/CSIRO DMS (Sydney), 1995. 86

29. P. Soille and J.-F. Rivest. Dimensionality of morphological operators and cluster analysis. In E. Dougherty, P. Gader, and J. Serra, editors, Image algebra and morphological image processing IV, volume 2030, pages 43-53. Society of PhotoInstrumentation Engineers, July 1993. 86

30. P. Soille and H. Talbot. Image structure orientation using mathematical morphology.In A. Jain, S. Venkatesh, and B. Lovell, editors, 14th International Conference on Pattern Recognition, volume 2, pages 1467-1469, Brisbane, August 1998. IAPR, IEEE Computer Society. 92

31. P. Soille and H. Talbot. Directional morphological filtering. IEEE Transactions on Pattern Analysis and Machine Intelligence, Submitted. 84, 92

32. S. Sternberg. Grayscale morphology. Computer Graphics and Image Processing, 35:333-355, 1986. 86

33. R. van den Boomgaard and R. van Balen. Methods for fast morphological image transforms using bitmapped binary images. Computer Vision, Graphics, and Image Processing: Graphical Models and Image Processing, 54(3):252-258, May 1992. 88

34. M. van Herk. A fast algorithm for local minimum and maximum filters on rectangular and octogonal kernels. Pattern Recognition Letters, 13:517-521, 1992. 82

35. P. Whelan, P. Soille, and A. Drimbarean. Real-time registration of paper watermarks. Real-Time Imaging, page In Press, 2000. 92 